\title{
Genetic Counselling and Genetic Screening for Alzheimer's Disease and Other Dementias
}

\author{
A. Dessa Sadovnick
}

\begin{abstract}
Genetic and nongenetic factors have been identified to have roles in the etiology of dementia. Etiologic heterogeneity and genetic heterogeneity are recognized, especially for Alzheimer's disease which is the most common form of dementia. Asymptomatic individuals are increasingly requesting genetic services such as genetic counselling, predictive testing and screening for genetic risk factors. This paper provides an overview of the current knowledge about genetic counselling and genetic screening for dementia as well as guidelines for the physician.
\end{abstract}

RÉSUMÉ: Conseil génétique et dépistage génétique de la maladie d'Alzheimer et des autres démences. Des facteurs génétiques et non génétiques ont été identifiés comme jouant un rôle dans l'étiologie de la démence. L'hétérogénéité étiologique et l'hétérogénéité génétique est reconnue, surtout pour la maladie d'Alzheimer qui est la forme la plus commune de démence. Les individus asymptomatiques font de plus en plus appel aux services de génétique comme le conseil génétique, le test prédictif et le dépistage des facteurs de risque. Cet article donne un aperçu des connaissances actuelles sur le conseil génétique et le dépistage génétique de la démence ainsi que des lignes directrices pour le médecin.

Can. J. Neurol. Sci. 2001; 28: Suppl. 1 - S52-S54

The term dementia refers to a heterogeneous group of diseases of which the most common is Alzheimer's disease (AD). Alzheimer's disease has an estimated incidence in Canada of 7.4/1000 women and 5.9/1000 men. ${ }^{1}$ Both genetic and nongenetic factors have roles in the "causation of" and "susceptibility to" dementia.

Dementia in general, and AD in particular are characterized by etiologic and genetic heterogeneity. In some families, a purely genetic cause can be identified and in others, an environmental cause. However, the majority of cases are multifactorial in nature, supporting a role for the interaction of genetic and environmental factors.

Dementia is a genetically complex group of disorders. Genetic heterogeneity is illustrated in AD by the presenilin 1 gene (PS1) located on chromosome 14q24.3 for which at least 60 mutations have been identified to date. ${ }^{2}$ Frontotemporal dementia (FTD) is similarly characterized by several mutations in the Tau coding region on chromosome 17 (FTD-17). ${ }^{3,4}$ Genetic susceptibility risk factors for AD have also been identified. The best documented of these is the apolipoprotein epsilon 4(apoE4)allele located on chromosome $19 q 13.2,{ }^{5-7}$ but others have also been proposed including the interleukin-1 (IL1) polymorphisms in IL-1Aand IL-1B genes. ${ }^{8,9}$

The awareness of the public that genetic factors have roles in the "cause of" or "susceptibility to" dementia has led to (and will probably increasingly lead to) requests by asymptomatic individuals for "genetic testing" and evaluation of risks for developing dementia. Nevertheless, despite ongoing research, ${ }^{10,11}$ there are no biological markers that are currently accepted for widespread use in predicting who will develop AD or other dementias. ${ }^{12}$ The only very rare exception is the option of testing for a family-specific genetic mutation ("Predictive Genetic Testing or "PGT") in an unaffected family member who is young enough to be "at risk" for the familial dementia.

Other than when PGT is an option, the dilemma facing clinicians is how to respond to the individual who asks about the likelihood that the individual or close family members will develop dementia in the future.

The following sections are designed to provide some practical guidelines for clinicians to whom such questions are addressed. The sections have been separated into the following:

(i) PGT is an option;

(ii) PGT is not an option at present but autosomal dominant inheritance is suspected (subdivided into early- and lateonset categories);

From the Department of Medical Genetics, University of British Columbia, Vancouver, BC Canada

Reprint requests to: A. Dessa Sadovnick, Department of Medical Genetics, University of British Columbia, G920, Detwiller Pavilion, Vancouver Hospital and Health Sciences Centre, UBC Site, 2211 Wesbrook Mall, Vancouver, BC, Canada V6T2B5 
(iii) There is a family history of dementia but this must be properly investigated before it can be determined whether or not there may be an autosomal dominant form of the dementia;

(iv) The family history is noncontributory but the asymptomatic individual is anxious;

(v) The role of genetic risk factors in predicting dementia;

(vi) Neuropathology;

(vii)Preventive measures.

When dealing with situations which fall into sections (i)-(iii), the clinician may wish to refer the individual to a genetics clinic. In Canada, most major cities have a university and/or hospital based facility which offers this service. [Information on regional clinical genetic services can be obtained through several sources including pediatric tertiary care hospitals, regional health units (e.g. in Thunder Bay, Ontario: www.tbdhu.on.ca) and the Canadian College of Medical Genetics (www.ccmg.medical.org). A new website for the Canadian Association of Genetic Counsellors will be available in 2001.

In Canada, a genetic counsellor often has a master's degree in the field and works as part of a clinical genetics team which can include, as necessary, clinical geneticists, molecular geneticists, medical ethicists and other health care professionals. The following is still one of the best available descriptions of genetic counselling: ${ }^{13}$ “. . . a communication process which deals with human problems associated with the occurrence, or risk of occurrence, of a genetic disorder in a family. This process involves an attempt by one or more appropriately trained persons to help the individual or the family to (1) comprehend the medical facts, including the diagnosis, the probable course of the disorder and the available management; (2) appreciate the way heredity contributes to the disorder and the risk of recurrence in specified relatives; (3) understand the options for dealing with the risk for recurrence; (4) choose the course of action which seems appropriate to them in view of their risks and their family goals and act in accordance with that decision; and (5) make the best possible adjustment to the disorder in an affected family member and/or to the risk of recurrence of that disorder".

\section{(i) PGT is an option}

In less than $5 \%$ of all families with dementia, it may be possible to identify a genetic mutation which appears to cause the family-specific dementia, e.g. a PS1 or tau mutation. These families tend to be characterized by early-onset dementia (well before age 65) which appears to follow an autosomal dominant mode of inheritance. In such situations, unaffected family members who are young enough to still be "at risk" for the familial dementia may have the option of PGT to determine whether or not they have inherited the family-specific mutation. In Canada, most genetics clinics will be able to test asymptomatic individuals for a known mutation under the "service mandate", i.e. the test is covered by the health plan. PGT includes several members of the genetics team since persons having this testing should clearly understand the various implications of such testing. Experience with PGT for Huntington's disease (and more recently for the BRCA-1 and BRCA-2 mutations in familial breast cancer $^{14}$ ) has shown the importance of pretest and posttest counselling when offering PGT. ${ }^{15}$ The issue of disclosure of family genetic information resulting from PGT to family and others has been the topic of consultative processes. ${ }^{16}$

PGT can alter an individual's a priori risk of up to $50 \%$ for the family specific type of dementia, based on the assumptions of the autosomal dominant model, to almost zero (mutation absent) or $100 \%$ (mutation present). It is, however, important to remember that the penetrance of identified mutations for $\mathrm{AD}$ and other dementias is not yet fully understood. ${ }^{17,18}$ In addition, in contrast to the situation for Huntington's disease ${ }^{15}$ but similar to that for breast cancer, ${ }^{14}$ the absence of a family-specific mutation does not protect that individual from AD or another dementia due to other causes. Phenocopies may also complicate PGT.

\section{(ii) PGT is not an option at present but autosomal dominant inheritance is suspected.}

In section (i) it is assumed that a family-specific mutation was identified before the asymptomatic individual seeks PGT. This is usually because other family members previously participated in a genetic research project. There are, however, other families with both early- and late-onset (over age 65) dementia where the mode of inheritance is compatible with autosomal dominant inheritance but no family-specific mutation has been identified. This presents a dilemma.

\section{Early-onset familial autosomal dominant dementia}

For the past decade, many research groups have been interested in identifying specific mutations in early-onset autosomal dominant dementia families. This enabled clinicians to enroll interesting families in a research protocol where affected individuals were screened for all known mutations and attempts were also made to identify new mutations. In the year 2000, this does not appear to be possible. Research interests have shifted and it is beyond the mandate of genetics "service" laboratories to conduct the complex analyses, including gene sequencing, needed to identify a mutation for the first time in a family. The only remaining options are private companies (local genetics clinics are aware of these). This is, however, very costly and is not covered by most Canadian health plans.

The recommended procedure, therefore, is to refer the family to a genetics clinic for counselling to discuss (i) the autosomal dominant mode of inheritance, (ii) advantages and limitations of trying to identify a mutation in the family for the first time and (iii) the option of DNA banking for affected family members as a safeguard for future developments.

If an unaffected family member from a suspected autosomal dominant pedigree is very anxious, it may be possible for a clinician to arrange detailed neuropsychological testing to provide a baseline for future comparison. However, in most instances, this may have to be paid for privately by the individual. Clinical neuropsychologists in a given region can be identified through psychology and neurology departments of local universities. The value of baseline screening with the Mini Mental State Examination ${ }^{19}$ is questionable. ${ }^{20}$

\section{Late onset familial autosomal dominant dementia}

Despite ongoing research, it has not yet been possible to identify a family-specific mutation for late-onset $\mathrm{AD}$, even if there is strong evidence for autosomal dominant inheritance. The recommended procedure is to refer the family to a genetics clinic for counselling to discuss the autosomal dominant mode of 
inheritance and the option of DNA banking as a safeguard for future developments. There are some research groups which may be interested in specific families, especially if at least three affected siblings are alive. These research groups can be reached through their publications using a Medline search.

Comments about neuropsychological baseline testing are the same as those discussed for early onset dementia.

\section{(iii) There is a family history of dementia but this must be properly investigated before it can be determined whether or not this is suggestive of autosomal dominant inheritance}

Individuals may state that "several family members" have dementia and give the impression that the family may represent autosomal dominant inheritance. However, as there are many causes of dementia, each reportedly affected family member must be investigated as thoroughly as possible to determine his/her "best estimate" dementia diagnosis. Often, by asking a few questions about the affected individuals, the clinician can readily determine that the dementia was due to a stroke, Parkinson's disease, or head trauma with resultant brain injury and therefore autosomal dominant inheritance of dementia is not likely. However, in some situations, more detailed follow-up (e.g. medical records, autopsy reports, etc.) is needed and this can be quite time consuming. Referral to a genetics clinic is appropriate as "documentation" of family history is part of the role of genetic counsellors.

If the clinician or genetic counsellor is comfortable that the family may represent an autosomal dominant form of dementia, the protocols outlined in sections (i) and (ii) should be followed as appropriate.

If it is determined that autosomal dominant inheritance of dementia is unlikely, counselling information can be provided as discussed in section (iv) below.

\section{(iv) The family history is noncontributory but the asymptomatic individual is anxious}

As stated earlier, the majority of individuals will not present to the clinician with a family history of dementia that is suggestive of autosomal dominant inheritance. In these instances, the unaffected individual is usually concerned about developing dementia in the future because there is an affected parent or sibling and/or a media report has recently focussed on dementia.

Having one affected first-degree relative can increase an individual's lifetime risk to develop dementia compared to the general population with no affected first degree relative. Risk counselling must be based on empiric data. In $\mathrm{AD}$, for example, life table analyses have shown that an asymptomatic individual from a family with at least one first-degree relative with AD has a lifetime risk to develop dementia which is approximately $3.5 \mathrm{X}$ the risk for "controls" (no known first-degree relative with dementia), e.g. $24 \%$ versus $7 \%$ by age 89 in British Columbia. ${ }^{21}$

If there is no affected first degree relative, an individual's risk to develop dementia is reflected by incidence data for the age and gender matched general population. ${ }^{1}$

Neuropsychological testing to establish a baseline is generally not offered.

(v) The role of genetic risk factors in predicting dementia

Universally applicable risk factors (genetic and nongenetic) for dementia must yet be determined. As stated earlier, at present the only undisputed genetic risk factor for dementia is the apoE4 allele. While apoE genotyping may have a role in the differential diagnosis of dementia, ${ }^{22,23}$ several publications, consensus statements and position papers have clearly stated that apoE genotyping should not be used for genetic testing for $\mathrm{AD}$ or other dementias in an asymptomatic individual. ${ }^{22-29}$

\section{(vi) Neuropathology}

The definitive diagnosis of AD and other types of dementia can often only be made after neuropathological examination. A definitive diagnosis is especially important if the family represents what is believed to be a purely genetic form of dementia. However, at present in Canada, availability of and eligibility criteria for neuropathology vary regionally. Clinicians are encouraged to contact local neuropathologists.

\section{(vii) Preventive measures}

Although no "preventive measures" including hormone replacement therapy, gingko biloba and anti-inflammatories are universally recommended at this time, ${ }^{30}$ this topic continues to be a focus of much research and the situation may change in the near future.

\section{SUMMARY (adapted from Sadovnick ${ }^{31}$ )}

1. For over $95 \%$ of families with dementia, there is no test or biomarker that can predict on an individual basis who will develop dementia in the future:

(i) clinicians should explain to asymptomatic individuals that no testing, including apoE genotyping, is available at present;

(ii)referral to a genetics clinic is usually not necessary.

2. If an autosomal dominant mode of inheritance is suspected, it is important to be able to assign the "best estimate" diagnosis of dementia for each reportedly affected family member. This can be done by the clinician or genetic counsellor, as appropriate. Documentation requiring medical records must be done with the informed consent of the appropriate family member (and/or next-of-kin/substitute decision-maker).

3. If an autosomal dominant mode of inheritance is supported after the "best estimate" diagnosis for each affected family member has been determined, referral to a genetics clinic is recommended.

4. If a family-specific genetic mutation has been identified, PGT may be an option for unaffected individuals. Referral to a genetics clinic is appropriate.

\section{REFERENCES}

1. Canadian Study of Health and Aging (CSHA) Working Group. CSHA: The incidence of dementia in Canada. Neurology 2000; 55:66-73.

2. Rosenberg RN. The molecular and genetic basis of AD: The end of the beginning. The 2000 Wartenberg lecture. Neurology 2000; 54:2045-2054

3. Froelich S, Basun H, Forsell C, et al. Mapping of a disease locus for familial rapidly progressive frontotemporal dementia to chromosome 17q12-21. Am J Med Genet (Neuropsychiatric Gen) 1997; 74:380-385.

4. Arima K, Kowalska A, Hasegawa M, et al. Two brothers with frontotemporal dementia and parkinsonism with an $\mathrm{N} 279 \mathrm{~K}$ mutation of the tau gene. Neurology 2000; 54:1787-1795. 
5. Corder EH, Saunders AM, Strittmatter WJ, et al. Gene dose of apolipoprotein E type 4 allele and the risk of Alzheimer's disease in late-onset families. Science 1993; 261:921-923.

6. Saunders AM, Strittmatter WJ, Schmechel D, et al. Association of apolipoprotein E allele E4 with late-onset familial and sporadic Alzheimer's disease. Neurology 1993; 43:1467-1472.

7. Poirier J, Davignon J, Bouthillier D, et al. Apolipoprotein E polymorphism and Alzheimer's disease. Lancet 1993; 342:697-699.

8. Grimaldi LM, Casadei VM, Ferri C, et al. Association of early-onset Alzheimer's disease with an interleukin- $1 \alpha$ gene polymorphism. Neurology 2000; 47:361-365

9. Nicoll JA, Mrak RE, Graham DI, et al. Association of interleukin-1 gene polymorphisms with Alzheimer's disease. Ann Neurol 2000; 47:365-368.

10. Ghanbari H, Ghanbari K, Beheshti I, et al. Biochemical assay for AD7C-NTPin urine as an Alzheimer's disease marker. J Clin Lab Anal 1998, 12:285-288.

11. Jeffries W, Feldman H, Nurminen J, et al. Serum p97 as a screening test for Alzheimer's disease. Neurobiol Aging 1998; 19(4s):S82.

12. The Ronald and Nancy Reagan Research Institute of the Alzheimer's Association and the National Institute on Aging Working Group. Consensus report of the working group on: "Molecular and biochemical markers of Alzheimer's disease". Neurobiol Aging 1998; 19:109-116.

13. Fraser FC. Genetic counselling. Am J Hum Genet 1974; 26:636659.

14. Claus EB, Schildkraut JM, Thompson WD, Risch NJ. The genetic attributable risk of breast and ovarian cancer. Cancer 1996; 77:2318-2324.

15. Wiggins $S$, Whyte $P$, Huggins $M$, et al. The psychological consequences of predictive testing for Huntington's disease. $\mathrm{N}$ Engl J Med 1992; 327:1401-1405.

16. ASHG Statement. Professional disclosure of familial genetic information. Am J Hum Genet 1998; 62:474-483.

17. Schellenberg GD, Payami H, Wijsman E, et al. Chromosome 14 and late-onset familial Alzheimer's disease (FAD). Am J Hum Genet 1994; 53:619-628.

18. Pericak-Vance MA, Grubber J, Bailey LR, et al. Genomic screen of 739 sibpairs with late-onset Alzheimer's disease. Am J Hum Genet 2000; 67(4):48.

19. Folstein MF, Folstein SE, McHugh PR. "Mini-mental-state": A practical method for grading the cognitive state of patients for the clinician. J Psychiatr Res 1975; 12:189-198.

20. Clark CM, Sheppard L, Fillenbaum GG, et al. Variability in annual Mini-Mental State Examination score in patients with probable Alzheimer's disease. Arch Neurol 1999; 56:857-862.

21. Hirst C, Sadovnick AD, Yee IML. Familial risks for Alzheimer's disease: data from an Alzheimer's clinic population. Genetic Epidemiol 1994; 11:365-374.

22. Bird TD. Apolipoprotein genotyping in the diagnosis of Alzheimer's disease: a cautionary view. Ann Neurol 1995; 38:2-4.

23. Roses AD. Apolipoprotein E genotyping in the differential diagnosis, not prediction, of Alzheimer's disease. Ann Neurol 1995; 38:6-14.

24. American College of Medical Genetics/American Society of Human Genetics Working Group on ApoE and Alzheimer's Disease. Statement on use of Apolipoprotein E testing for Alzheimer's disease. J Am Med Assoc 1995; 274:1627-1629.

25. Blacker D, Haines JL, Rodes L, et al. ApoE-4 and age at onset of Alzheimer's disease: The NIMH Genetics Initiative. Neurology 1997; 48:139-147.

26. National Institute on Aging/Alzheimer's Association Working Group. Apolipoprotein E genotyping in Alzheimer's disease. Lancet 1996; 347:1091-1095.

27. Post SG, Whitehouse PJ, Binstock RH, et al. Consensus statement: the clinical introduction of genetic testing for Alzheimer's disease. JAMA1997; 277:832-840.

28. Kalaria RN, Giacobini E. Meeting Report: The Fifth International Conference on Alzheimer's Disease and related Disorders. Alzheimer Dis Assoc Disord 1997; 11:113-119.

29. Blacker N. New insights into genetic aspects of Alzheimer's disease. Does genetic information make a difference in clinical practice? Postgrad Med 2000; 108:119-122.

30. Patterson CJS, Gauthier S, Bergman H, et al. The recognition and management of dementing disorders: conclusions from the Canadian Consensus Conference on Dementia. Can Med Assoc J 1999; 160 (supplement 12).

31. Sadovnick AD. Genetic counselling in dementia. In: Qizilbash N, Schneider L, Chui H, Tariot P, Brodaty H, Kaye J, Erkinjuntti T eds. Evidence-Based Dementia: A Practical Guide to Diagnosis and Management (with internet updates). Oxford: Blackwells. In press, 2001. 